\title{
G-CSF guideline adherence in Germany, an update with a retrospective and representative sample survey
}

\author{
Hartmut Link ${ }^{1} \cdot$ Markus Kerkmann ${ }^{2} \cdot$ Laura Holtmann $^{2} \cdot$ Petra Ortner $^{3} \cdot$ for the Working Groups Supportive Care \\ (ASORS now AGSMO) and Medical Oncology (AIO) within the German Cancer Society (DKG)
}

Received: 22 March 2018 / Accepted: 18 September 2018 / Published online: 29 October 2018

(C) The Author(s) 2018

\begin{abstract}
Background Current guidelines (GL) recommend neutropenia prophylaxis with G-CSF after chemotherapy (CTX) for patients with high $(\geq 20 \%)$, or, if additional risk factors are present, intermediate $(\geq 10-20 \%)$ risk of febrile neutropenia. The first sample survey in 2012 (NP1) showed lack of GL adherence. The aim of this second sample survey was to evaluate if GL adherence and implementation have improved.

Methods The sample size represented $1.0 \%$ of the incidences of lung and $1.1 \%$ of breast cancer in Germany in 2010. Data of patients with a febrile neutropenia (FN) risk $\geq 10 \%$ who had received at least 2 cycles of chemotherapy between October 2014 and September 2015 was surveyed retrospectively.

Results Data from 573 lung cancer (LC) and 801 breast cancer (BC) patients was collected from 109 hospitals and 83 oncology practices with 222 physicians participating. Compared with the NP1 survey, GL adherence increased in LC and FN high-risk (HR) chemotherapy from 15.4 to $47.8 \%(p<0.001)$, and in FN intermediate-risk (IR) chemotherapy from 38.8 to $44.3 \%$ ( $p=$ 0.003). In BC and FN-HR chemotherapy, GL adherence was unchanged: $85.6 \%$ vs. $85.1 \%$ ( $p=0.73$ ) but increased in FN-IR from 49.3 to $57.8 \%(p<0.001)$. In all IR CTX cycles, there are also no significant differences in GL adherence between the first $(51.3 \%)$ and subsequent cycles $(51.1 \% ; p=0.948)$. In LC patients treated in certified or comprehensive cancer centers, the GL adherence in FN-HR chemotherapy was $53.0 \%$ vs. $44.9 \%$ in other centers $(p=0.295)$; in FN-IR chemotherapy, it was $45.1 \%$ vs. $43.8 \%(p=0.750)$. In BC with FN-HR chemotherapy, GL adherence in certified or comprehensive centers was $85.4 \%$ vs. $84.7 \%$ in other institutions $(p=0.869)$; in FN-IR chemotherapy, it was $60.2 \%$ vs. $55.0 \%(p=0.139)$. GL adherence in FN-HR chemotherapy and in FN-IR chemotherapy differed between pulmonologists and hematologist-oncologists (FN-HR: $25.0 \%$ vs. $43.6 \%$, $p<0.001 ; 38.1 \%$ vs. $48.6 \%, p<0.001)$. Comparing gynecologists with hematologist-oncologists, GL adherence in FN-HR chemotherapy was $86.2 \%$ vs. $82.5 \%$. In FN-IR chemotherapy, GL adherence by gynecologists and hematologist-oncologists was $58.6 \%$ and $55.6 \%$, respectively $(p=0.288 ; p=0.424)$. Classification and regression tree analysis split pulmonologists and other specialists, with the latter adhering more to GL $(p<0.001)$. Hematologist-oncologists and gynecologists with more than 2 years of professional training in medical cancer therapy adhered more closely to GL than others $(68.7 \%$ vs. $46.2 \%, p<0.001)$. Pulmonologists attending $\geq 2$ national congresses annually adhered more to guidelines than other pulmonologists ( $44.8 \%$ vs. $24.3 \%, p<0.001)$.

Conclusions Adherence to G-CSF GL in Germany has increased but is still insufficient. Certified and comprehensive cancer centers show a higher rate of GL implementation. In GL adherence, there is still a disparity between cancer types and between oncology treatment specialists.
\end{abstract}

Keywords Breast cancer · Lung cancer · G-CSF-prophylaxis · Febrile neutropenia · G-CSF guideline · Guideline adherence

Hartmut Link

hlink@kabelmail.de
2 MMF GmbH, Heideblick 59, 44229 Dortmund, Germany

3 POMME-med GmbH, Von-Erckert-Str. 48, 81827 Munich, Germany

1 Internal Medicine, Hematology and Medical Oncology, Kaiserslautern, Pfaffplatz 10A, 67653 Kaiserslautern, Germany 


\section{Introduction}

Use of hematopoietic growth factors is the recommended standard of care to prevent neutropenia and febrile neutropenia in patients with malignant diseases undergoing chemotherapy. The aim of neutropenia prophylaxis is to prevent neutropenic infections, which manifest as febrile neutropenia and can potentially be lethal $[1,2]$. Neutropenia-associated complications often lead to dose reductions or delays in chemotherapy and thus to a reduced relative dose intensity, which can worsen the survival of patients [3]. The rate of infection-associated complications should therefore be kept low.

Consistent prophylaxis of neutropenia and febrile neutropenia with the hematopoietic growth factors G-CSF or GMCSF for the stimulation of granulopoiesis directly after chemotherapy is therefore indispensable. The application of these factors is defined by guidelines based on evidence from clinical trials [4-10]. It has recently been published that insufficient guideline adherence increases the risk of problems caused by chemotherapy-induced neutropenia and febrile neutropenia [11].

A first study in 2012 showed that the implementation of guidelines for neutropenia prophylaxis with G-CSF in Germany was not sufficient [12]. This is why many training courses, workshops, lectures, and conferences have taken place to highlight the problem of inadequate guideline adherence. A second representative study has now been carried out in 2015 to determine whether progress has been made in the implementation of the G-CSF prophylaxis guidelines.

\section{Aims of the study}

The primary aim of the study is to examine the implementation of the updated European Organisation for Research and Treatment of Cancer (EORTC) guideline (2010) on primary prophylaxis with G-CSF in patients who have undergone a chemotherapy that is associated with a high $(>20 \%)$ or intermediate (10-20\%) risk of febrile neutropenia (FN).

To this end, a nationwide representative survey was conducted into the current practice of neutropenia prophylaxis in medical practices and hospitals in the case of two relevant oncological tumor entities in which chemotherapies with a high or intermediate FN risk are frequently used: breast and lung cancer. The results were compared with those of the first nationwide representative survey in 2011/12 ("neutropenia prophylaxis I") to establish to what extent and in what areas adherence to the guideline in Germany has improved over recent years [12].

In addition, the attending physicians were surveyed about their competence profile, their assessment of the quality of the guidelines, and their approach to neutropenia prophylaxis. The aim was to determine whether and to what degree this correlates with the guideline-compliant therapy. These results were also compared with the data from the first quality assurance initiative (neutropenia prophylaxis I).

A further aim was to examine the extent to which the following certified centers differ from other establishments in their adherence to the guidelines. This was based on the following certifications: breast cancer center (German Cancer Society - DKG), lung cancer center (DKG), oncological center (DKG), oncological center (German Society for Hematology and Medical Oncology - DGHO), and comprehensive cancer center (CCC, DKG).

\section{Hypotheses}

This study sought to explore the implementation of guidelines on neutropenia prophylaxis in day-to-day treatment in Germany. As the main hypothesis, it is assumed that implementation of the guidelines has improved compared with the first quality assurance initiative (2011/12).

- The implementation of guidelines on neutropenia prophylaxis differs along the treatment structure

- The competence profile of the attending physicians correlates with guideline-compliant neutropenia prophylaxis.

\section{Methods}

This was a retrospective sample survey representative for German hospitals and practices applying chemotherapy in patients with breast cancer or lung cancer. To achieve a reliable sample, which is representative for Germany, the distribution of the patients to be documented was specified in the individual indications among the facilities involved. This was done using the facilities' data on patient numbers and treatment structure from the first nationwide representative survey in 2011/12 (neutropenia prophylaxis I) [12]. The participating centers are assigned to clusters based on key distinguishing features (facility type, care level, specialization, and number of patients treated) and their percentage of the sample is determined by comparison with the data from the health care structure analysis of 2012. By taking this approach, the actual percentages of the various care facilities in an indication area can be reflected as proportionally as possible in the patient documentation sample. In order to address the problem of inflation of type I errors (false-positive or $\alpha$-errors) by multiple testing, the $p$ values were adjusted using the Benjamini and Hochberg procedure to control the false discovery rate (FDR) [13]. Since the design of this study is explorative in character, correction of the FDR is more appropriate than a Bonferroni-based correction of the family-wise error rate 
(FWER). Although FWER correction is strong in controlling type I errors, it ignores dependencies among the data and is also associated with inflation of type II errors (false-negative or $\beta$-errors), thus existing correlations may not be identified due to overcorrection [14]. The sample size was set at approximately $1 \%$ of the annual incidence of each new diagnosis in Germany to obtain reliable and valid analytical results, i.e., 800 patients with breast and 600 with lung cancer. Data was collected completely anonymously so that neither the attending physician nor the institute-commissioned AIO or ASORS could identify or trace back patients from the personal data after it had been recorded. Statistical analyses were performed with the SPSS 19 statistical package. As far as applicable, the study was compliant with the STROBE guidelines [15]. To describe possible links between guideline adherence and the professional competence profile of the physicians, a classification and regression tree (CART) analysis was performed. CART is a tree-building binary recursive partitioning method using the Gini index for discrete distributions [16, 17]. Details of this method have been published previously [12]. For each chemotherapy cycle, compliance with the defined standard was taken as basis. The following factors were included in this analysis: age; academic degree; specialist training; length of training in oncology; duration of training in drug tumor therapy; position in the practice/department; specialization; activity in study groups; publications in scientific journals and textbooks; active participation in guidelines and guidelines within the clinic or practice; participation in regional, national, and international congresses; participation in further training; and participation in quality assurance. In addition, to control and validate the results of the CART algorithm, the calculated subgroups were compared by calculating the relative risk (RR) and Pearson's chi-square test for discrete distributions.

\section{Patients}

Patients must have received at least 2 cycles of a chemotherapy associated with high or intermediate FN risk (according to current EORTC guidelines) between 1 October 2014 and 31 March 2015 [8]. The list of the chemotherapy protocols is given in Table 1. The FN risk has been taken from the original publications, which are also used in the German online database of chemotherapy protocols "Onkopti®” (www.onkopti.de).

At least two and a maximum of three chemotherapy cycles per patient were to be documented. The restriction to the number of 3 cycles is due to the results of the initial study, neutropenia prophylaxis I, in which no changes in the treatments over the further cycles could be detected [12].

The investigated chemotherapy regimens associated with high or intermediate risk for febrile neutropenia are commonly used in Germany. Potential minor variations within the protocols were evaluated by the scientific project lead and assigned to the corresponding FN risk.

Primary prophylaxis with G-CSF after chemotherapy is recommended if the risk of $\mathrm{FN}$ due to chemotherapy is high $(>20 \%)$. If the risk of $\mathrm{FN}$ is intermediate $(\geq 10-20 \%)$, then such prophylactic treatment should be given if additional risk factors are present.

\section{The risk factors}

At least one of the following risk factors for FN: age > 65 years, advanced stage of disease, prior febrile neutropenia due to chemotherapy; or at least two of the following risk factors for FN: poor performance status (ECOG > 1), hemoglobin $<12 \mathrm{~g} / \mathrm{dl}$, liver, renal, or cardiovascular disease, previous non-febrile neutropenia, female gender; or at least three other comorbid conditions. Poor nutrition status is mentioned by the EORTC guidelines as well; however, it was noted only in very few patients and thus not included for this grouping.

\section{Results}

The data of 1415 patients was collected; 602 with lung cancer and 813 with breast cancer. Data of 41 patients did not match the inclusion criteria, so a total of 1374 patients were included in the study, 573 with lung cancer and 801 with breast cancer, who received 1620 and 2332 chemotherapy cycles, respectively. One hundred twenty-five clinics and 97 practices with a total of 222 physicians participated.

\section{Guideline adherence compared to the 2012 study}

Compared with the first quality assurance for neutropenia prophylaxis (NP1) of 2012, the overall guideline adherence has improved significantly. Over both observed indications, compliance with the standard has improved from 65.1 to $76.6 \%$ ( $p<0.001$; OR 1.76; 95\% CI 1.50-2.07) for chemotherapy with high FN risk, and from 45.5 to $51.2 \%$ for chemotherapy with intermediate FN risk $(p<0.001$; OR 1.26; 95\% CI 1.14 1.38).

The most significant improvement was seen in the treatment of lung cancer with chemotherapy with a high FN risk, where G-CSF treatment in accordance with the guidelines has increased from 15.4 to $47.8 \%$ ( $p<0.001$; OR 5.06; $95 \%$ CI 3.66-6.99). Chemotherapy with intermediate FN risk also showed an improvement of 38.8 to $44.3 \%$, although this is more moderate $(p=0.004$; OR 1.26 ; $95 \%$ CI 1.08 1.46).

In the treatment of breast cancer, compliance with the standard has remained constant at a high level in chemotherapy with a high risk of FN: $85.6 \%$ (2012) vs. $85.1 \%$ (2015), 
Table 1 High and intermediate risk regimens for lung and breast cancer patients; the numbers correspond to the dosages per square meter of the substances. AUC = area under the curve for dosing of carboplatin according the Calvert formula. Data originates from the original publications and is mainly published by the German database for oncological treatment protocols Onkopti ${ }^{\circledR}$, www.onkopti.de; $\mathrm{dd}=$ dose dense
Lung cancer (NSCLC)

Gemcitabine 1000/carboplatin 5 AUC

Vinorelbine 30/cisplatin 100 (palliative)

Gemcitabine 1250/carboplatin 5 AUC

Gemcitabine 1200/carboplatin 5 AUC

Lung cancer (SCLC)

Topotecan $1.5(\mathrm{~d} 1-5)$

Carboplatin 5 AUC/etoposide 140

Cisplatin 100/etoposide 100

Doxorubicin 60/cyclophosphamide 750/vincristine 1.5 (ACO II)

Epirubicin 40/cyclophosphamide 750/vincristine 1.5 (EPICO)

Cisplatin 25 (d1-3)/etoposide 80 (d1-3)

Etoposide $50 \mathrm{~d} 1-21(\mathrm{q} 4 \mathrm{w})$

Breast cancer

Docetaxel 75/doxorubicin 50/cyclophosphamide (TAC) 500

Epirubicin $150 \rightarrow$ paclitaxel $225 \rightarrow$ cyclophosphamide 2500 (ETC) (dd)

dd Epirubicin 90/cyclophosphamide $600 \rightarrow$ paclitaxel 175 (q2w)

Docetaxel 75/carboplatin 6 AUC (TCH)

dd Epirubicin $150 \rightarrow$ nab-paclitaxel $330 \rightarrow$ cyclophosphamide 200

(GAIN-2 study)

dd Epirubicin 90/cyclophosphamide $600 \rightarrow$ docetaxel 60 (EC-part) (q2w)

Epirubicin $150 \rightarrow$ paclitaxel $225 \rightarrow$ cyclophosphamide $2000(\mathrm{q} 2 \mathrm{w})$

(GeparOcto study)

Paclitaxel 175 (mono) (q2w)

Doxorubicin 60/cyclophosphamide $600 \rightarrow$-paclitaxel 175 (q2w)

(AC-part)

Docetaxel 75/cyclophosphamide 600

dd Epirubicin 90/cyclophosphamide $600 \rightarrow$ docetaxel 75 (q2w)

(Doce-Part)

Doxorubicin 75/cyclophosphamide 600

Docetaxel 100 (with trastuzumab)

Doxorubicin $60 \rightarrow$ paclitaxel $175 \rightarrow$ cyclophosphamide 600

$(\mathrm{A} \rightarrow \mathrm{P} \rightarrow \mathrm{C})$ (q2w)

Epirubicin 75/cyclophosphamide 600 (q2w)

Doxorubicin 60/cyclophosphamide $600 \rightarrow$ paclitaxel 175 (q2w) (Pac-part)

Doxorubicin 50/docetaxel 75
Carboplatin 5 AUC/etoposide 100

Vinorelbine 30/cisplatin 80

Vinorelbine 25/cisplatin 100

Paclitaxel 200/carboplatin 6 AUC (with bevacizumab)

Paclitaxel 200/carboplatin 6 AUC (consolidation after radiotherapy)

Docetaxel 75 (mono)

Vinorelbine 30/cisplatin 100 (adjuvant)

Docetaxel 75 (with nintedanib)

Vinorelbine 30/gemcitabine 1200 (d1.8 q3w)

Vinorelbine 25/carboplatin 5

Docetaxel 75/cisplatin 75

Docetaxel 75/carboplatin 6 AUC

Cisplatin 100/etoposide 100

Docetaxel 85/gemcitabine 1000

Carboplatin 5 AUC/etoposide 100

Cisplatin 75/etoposide 100

Carboplatin 5 AUC/etoposide 120 (q3w)

Cisplatin 80/etoposide 120 (q4w)

Cisplatin 80/etoposide 100

Cisplatin 90/etoposide 150

Cisplatin 80/etoposide 120 (q3w)

Carboplatin 6 AUC/etoposide 120 (q4w)

Carboplatin 300/etoposide 100

Cisplatin 60/etoposide 120

Cyclophosphamide 1000/doxorubicin 50/Vincristine 1,4 (CAV)

Carboplatin 5 AUC/etoposide 80

Cisplatin 80/etoposide 150

Carboplatin 300 (d1)/etoposide 140 (d1-3)/vincristine 1.4 (d1.8.15)

Topotecan $1.5 /$ cisplatin 75

Topotecan 2.3

Topotecan $1.5(\mathrm{~d} 1-4)$

Topotecan $0.75(\mathrm{~d} 1-5) /$ carboplatin 5 AUC

Irinotecan 60/cisplatin 60

Cisplatin 20/etoposide 75/ifosfamide 1,2 (d1-4 q3w)

Cisplatin 80/etoposide 80

Epirubicin 90/cyclophosphamide 600 (q3w)

Epirubicin 75/cyclophosphamide 600 (q3w)

Doxorubicin 75/cyclophosphamide 600 (q3w)

Vinorelbine 30 (with trastuzumab)(d1,8 q3w)

Docetaxel 100 (with trastuzumab, pertuzumab)

Eribulin 1.23 d1.8

Docetaxel 75 (with trastuzumab, pertuzumab)

Doxorubicin 60/cyclophosphamide $600 \rightarrow$ paclitaxel 80 weekly (AC-part) (q3w)

5-Fluoruracil 500/epirubicin 90/cyclophosphamide 600 (FEC) $\rightarrow$ docetaxel 100 (Doce-part)

Epirubicin 90/cyclophosphamide $600 \rightarrow$ docetaxel 60 (Doce-part) (q3w)

Gemcitabine 1000/carboplatin 4 AUC

Docetaxel 75/gemcitabine 1000

Doxorubicin (lip.) 75/cyclophosphamide 600

Paclitaxel 175 (mono) (q3w)

Paclitaxel 175 (mono) (q4w)

Gemcitabine 1000/cisplatin $50 \mathrm{~d} 1.14$ 
so that no significant differences can be observed ( $p=0.726$; OR $0.96 ; 95 \%$ CI $0.76-1.21$ ). An improvement of 49.3 to $57.8 \%$ can be observed in chemotherapy regimens with intermediate FN risk $(p<0.001$; OR 1.40; 95\% CI 1.23-1.60).

\section{Guideline adherence in certified and non-certified centers}

There are slight differences in compliance with the standard between certified and non-certified centers (OnkoZert/DKG, DGHO, CCC) (see Figure 1 and Table 2). The difference that can be observed across both indications is also due to the fact that in the indication of breast cancer, the overall guideline adherence is higher and a greater proportion of patients is treated in certified centers so that the summary shows a statistically significant result observed over both indications (see Fig. 1).

\section{Guideline adherence and specialties of the centers}

There are significant differences between the two organspecific disciplines (pulmonology and gynecology) and hematology/oncology institutions. In pulmonology, adherence to the standard is significantly lower than in hematology/ oncology departments or practices: in chemotherapy protocols with a high FN risk, 25.0\% in pulmonology departments and $43.6 \%$ in hematology/oncology departments or practices adhere to the standard ( $p<0.001$; OR $0.26 ; 95 \%$ CI $0.14-0.46)$. In the case of chemotherapy protocols with intermediate FN risk, $38.1 \%$ in pulmonology vs. $48.6 \%$ in hematology/ oncology adhere to the standard $(p<0.001$; OR $0.58 ; 95 \%$ CI 0.47-0.72).

In gynecology, there is a trend towards greater guideline compliance in gynecology departments and practices compared with hematology/oncology departments and practices. However, this difference is not statistically significant in either high or intermediate FN risk chemotherapy. In the case of chemotherapy protocols with a high FN risk, $86.2 \%$ of the gynecological institutions adhere to the standard vs. $82.5 \%$ in hematology/oncology $(p=0.288$; OR 1.37 ; $95 \%$ CI 0.90 1.93). For protocols with intermediate FN risk, the standard is met in gynecology in $58.6 \%$ of the cycles, in hematology/ oncology in $55.6 \%$ of the cycles ( $p=0.424$; OR 1.13 ; $95 \%$ CI 0.89-1.43).

There are no differences between hospital- and officebased physicians in the case of chemotherapy protocols with high FN risk. Across both indications, adherence to guidelines (GL) is $75.4 \%$ vs $78.0 \%$, respectively, $p=0.277$; OR 0.86 ; 95\% CI $0.66-1.13$ ). In lung cancer (LC), it is $44.2 \%$ vs. 53. $0 \%(p=0.288$; OR $0.70 ; 95 \%$ CI $0.43-1.13)$; in breast cancer (BC), it is $86.0 \%$ vs $84.2 \%$ ( $p=0.288$, OR 1.15 ; $95 \%$ CI 0.80 1.65).
However, in the case of chemotherapy protocols with intermediate FN risk, there are some differences between hospitals and office-based physicians: across both indications, GL adherence was $47.6 \%$ vs. $57.1 \%$ ( $p<0.001$, OR $0.68 ; 95 \%$ CI $0.59-0.80$ ); in LC, it was $40.1 \%$ vs. $56.3 \%$ ( $p<0.001$; OR $0.52 ; 95 \%$ CI $0.41-0.67)$. In contrast, there was no difference in BC: $58.0 \%$ vs. $57.5 \%$ ( $p=0.869$; OR 1.02 ; $95 \%$ CI $0.83-$ 1.27).

\section{Comparison of guideline adherence between the first and subsequent cycles}

In FN high-risk regimes ( $>20 \%)$, there are no significant differences between the first and subsequent cycles: all indications (first cycle $74.4 \%$ vs. $77.8 \%$ in subsequent cycles; $p=$ 0.298 ; OR $=1.202 ; 95 \%$ CI 0.913-1.584); lung cancer (first cycle $47.0 \%$ vs. $48.3 \%$ in subsequent cycles, $p=0.869$; OR $1.054 ; 95 \% \mathrm{CI}=0.645-1.722$ ) and breast cancer (first cycle $83.0 \%$ vs. $86.2 \%$ in subsequent cycles; $p=0.298$; OR 1.275 ; 95\% CI 0.880-1.847).

In FN intermediate-risk regimes (10-20\%), there are also no significant differences between first $(51.3 \%)$ and subsequent cycles $(51.1 \% ; p=0.948 ; \mathrm{OR}=0.995 ; 95 \%$ CI 0.850 1.164). Nonetheless, in lung cancer, GL adherence is slightly higher in subsequent cycles $(46.1 \%)$ than in first cycles $(41.0 \%)(p=0.168$; OR 1.125 ; 95\% CI $0.982-1.546)$, whereas in breast cancer, the GL adherence is somewhat lower in subsequent cycles $(55.9 \%)$ than in first cycle $(61.3 \%$; $p=$ 0.139 ; OR 0.800 ; $95 \%$ CI $0.639-1.002$ ). The data is summarized in Table 2. Table 3 shows that GL adherence in LC in the second cycle is often associated with dose reduction, but dose reduction plays a minor role in $\mathrm{BC}$.

\section{Are the costs an argument against G-CSF prophylaxis?}

In this study, $78.4 \%$ of physicians stated that the costs did not play a role in deciding on G-CSF prophylaxis, while 13.5\% said they did not prescribe G-CSF for cost reasons.

\section{Self-perception, self-assessment of the doctors}

In this study, $81.1 \%$ of the physicians interviewed stated that they regularly apply guidelines on chemotherapy, $74.8 \%$ of physicians indicated that they regularly apply guidelines, and $16.2 \%$ apply guidelines for G-CSF prophylaxis in unclear situations.

\section{Importance of the recommendations and professional journals on G-CSF prophylaxis}

Physicians were asked which guidelines they considered relevant for their decision regarding G-GSF prophylaxis. In addition to guidelines from specialist societies and scientific 
$\square$ standard met $\square$ standard not met

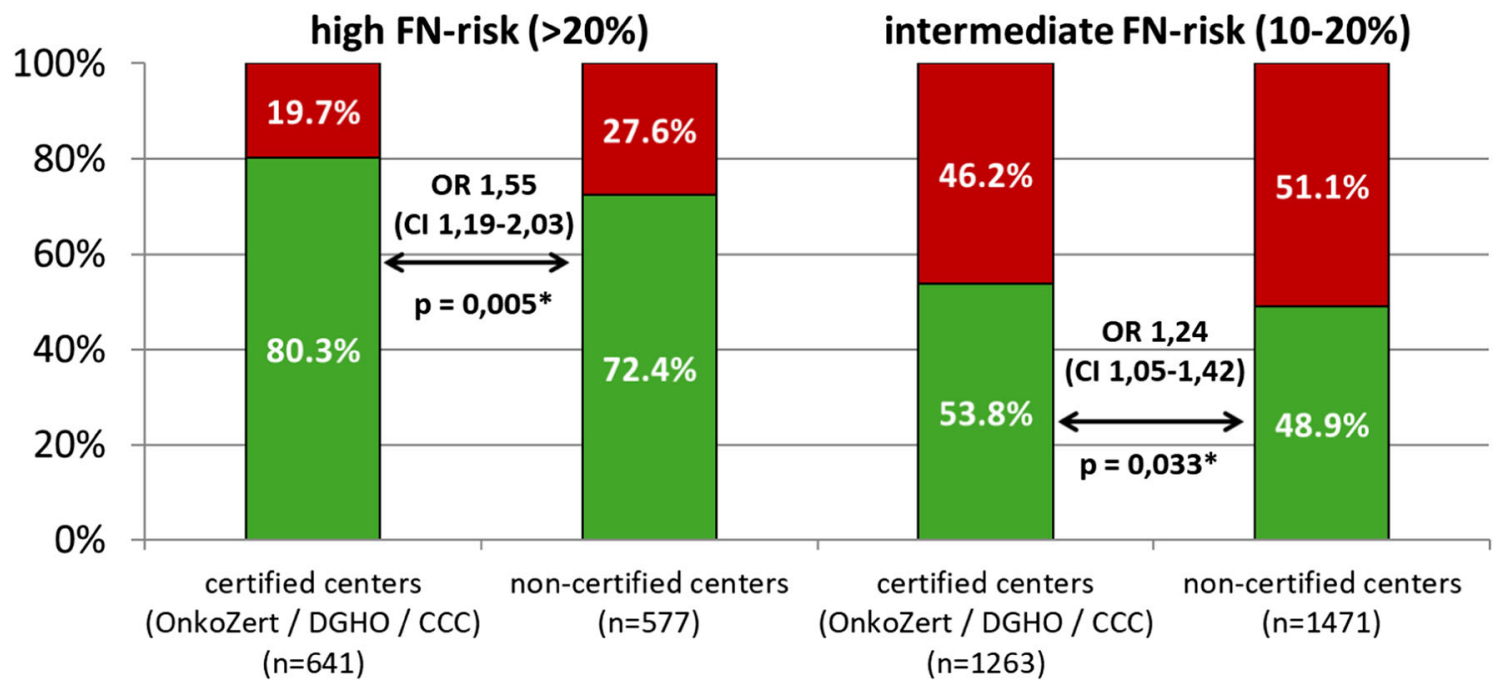

Fig. 1 Adherence to EORTC and ASCO G-CSF guidelines to reduce the incidence of febrile neutropenia after chemotherapy by center certification (OnkoZert/DGHO/CCC). FN febrile neutropenia; OR odds ratio;

Pearson's chi-squared test, $p$ values adjusted using the Benjamini and Hochberg procedure to control the false discovery rate (FDR)

studies, experience provides an important basis for decisionmaking (see Fig. 2). In this study, $82.4 \%$ of physicians stated that they read scientific journals on supportive therapy and $91 \%$ on drug therapy (see Fig. 3).

\section{Guideline adherence depending on the competence profile of the physicians}

In order to investigate the possible relations between the treatment in accordance with the guidelines and the competence profile of the treating physicians, the data of the patient documentation and the physician survey were correlated and analyzed with classification and regression tree (CART), the methodological details were published in the study Neutropenia I 2012 [12].

Among the factors defined for describing the professionalism of the physicians, the first split appeared between pulmonologists on one side, and hematologists/oncologists and gynecologists on the other, with higher GL adherence in the latter two. Within the group of pulmonologists, GL adherence was better if they participated in two or more national conferences per year.

Gynecologists and hematologists/oncologists showed higher GL adherence when they had professional experience of more than 2 years. Physicians with 2 years or less of professional training was split by the specialization in therapy of patients with gynecological tumors (better adherence of $70.5 \%$ ) or no specialization and treating mainly lung cancer (less adherence of 52.1\%). The results are depicted in Fig. 4.

\section{Discussion}

The study shows that the prophylaxis of febrile neutropenia in Germany has improved in the years 2012 to 2015 for the selected indications breast cancer and lung cancer [12]. It can therefore be assumed that the awareness of the treating oncologists has changed and that the guideline awareness has increased. The publication of the first study, with disappointing results of guideline adherence, as paper and on seminars, congresses and training events, for example, probably contributed to this. However, there are considerable differences between fields of expertise. Overall, the gynecological oncologists are more guideline-adherent than hematologist-oncologists and pulmonologists.

In lung cancer, the guideline coherence has improved compared to the preliminary examination, but it is still far too low. Compliance with the standard is less than $50 \%$ for both high and intermediate FN risk chemotherapy.

It is known that in advanced lung cancer, $25 \%$ of the patients take a fatalistic attitude [18]. This could also motivate doctors to administer less stringent chemotherapy and supportive therapy. However, it is not possible to explain why patients receive a dose-reduced therapy, with an increased risk of complications, without the appropriate guideline recommendation of G-CSF prophylaxis, instead of an established, full-dose primary therapy with adequate G-CSF prophylaxis which has proven effective in multiple studies. It has been published that the assessment of FN risk factors by physicians varies. Besides the risk of chemotherapy, the assessment of the patient by the physician plays a decisive role in risk 
Table 2 Guideline adherence in certified and non-certified centers

\begin{tabular}{|c|c|c|c|c|c|c|}
\hline & Indication & $\begin{array}{l}\text { Standard met } \\
\text { in certified } \\
\text { centers }(\%)\end{array}$ & $\begin{array}{l}\text { Standard met in } \\
\text { non-certified } \\
\text { centers }(\%)\end{array}$ & $p$ value & $\begin{array}{l}\text { Odds } \\
\text { ratio }\end{array}$ & $\begin{array}{l}95 \% \text { confidence } \\
\text { interval }\end{array}$ \\
\hline \multirow[t]{3}{*}{ High FN risk } & Both indications & 80.3 & 72.4 & 0.005 & 1.55 & $1.19-2.03$ \\
\hline & Lung cancer & 53.0 & 44.9 & 0.296 & 1.38 & $0.85-2.26$ \\
\hline & Breast cancer & 85.4 & 84.7 & 0.869 & 1.06 & $0.74-1.52$ \\
\hline \multirow[t]{3}{*}{ Intermediate $\mathrm{FN}$ risk } & Both indications & 53.8 & 46.2 & 0.033 & 1.24 & $1.05-1.42$ \\
\hline & Lung cancer & 45.1 & 43.8 & 0.750 & 1.06 & $0.85-1.31$ \\
\hline & Breast cancer & 60.2 & 55.0 & 0.139 & 1.24 & $1.00-1.53$ \\
\hline \multicolumn{7}{|c|}{ Guideline adherence and specialties of the centers } \\
\hline & Indication & $\begin{array}{l}\text { Standard met in } \\
\text { organ-specific } \\
\text { (pulmonological/ } \\
\text { gynecological) } \\
\text { departments }(\%)\end{array}$ & $\begin{array}{l}\text { Standard met in } \\
\text { hematological/ } \\
\text { oncological } \\
\text { departments }(\%)\end{array}$ & $p$ value & $\begin{array}{l}\text { Odds } \\
\text { ratio }\end{array}$ & $\begin{array}{l}95 \% \text { confidence } \\
\text { interval }\end{array}$ \\
\hline \multirow[t]{2}{*}{ High FN risk } & Lung cancer & 25.0 & 43.6 & $<0.001$ & 0.26 & $0.14-0.46$ \\
\hline & Breast cancer & 86.2 & 82.5 & 0.288 & 1.37 & $0.90-1.93$ \\
\hline \multirow[t]{3}{*}{ Intermediate FN risk } & Lung cancer & 38.1 & 48.6 & $<0.001$ & 0.58 & $0.47-0.72$ \\
\hline & Breast cancer & 58.6 & 55.6 & 0.424 & 1.13 & $0.89-1.43$ \\
\hline & Indication & $\begin{array}{r}\text { Standard met in } \\
\text { hospitals }(\%)\end{array}$ & $\begin{array}{l}\text { Standard met in } \\
\text { office-based } \\
\text { physicians (\%) }\end{array}$ & $p$ value & $\begin{array}{l}\text { Odds } \\
\text { ratio }\end{array}$ & $\begin{array}{l}95 \% \text { confidence } \\
\text { interval }\end{array}$ \\
\hline \multirow[t]{3}{*}{ High FN risk } & Both indications & 75.4 & 78.0 & 0.277 & 0.86 & $0.66-1.13$ \\
\hline & Lung cancer & 44.2 & 53.0 & 0.288 & 0.70 & $0.43-1.13$ \\
\hline & Breast cancer & 86.0 & 84.2 & 0.288 & 1.15 & $0.80-1.65$ \\
\hline \multirow[t]{3}{*}{ Intermediate FN risk } & Both indications & 47.6 & 57.1 & $<0.001$ & 0.68 & $0.59-0.80$ \\
\hline & Lung cancer & 40.1 & 56.3 & $<0.001$ & 0.52 & $0.41-0.67$ \\
\hline & Breast cancer & 58.0 & 57.5 & 0.869 & 1.02 & $0.83-1.27$ \\
\hline \multicolumn{7}{|c|}{ Comparison of guideline adherence between the first cycle and subsequent cycles } \\
\hline & Indication & $\begin{array}{l}\text { Standard met in } \\
\quad \text { first cycle }(\%)\end{array}$ & $\begin{array}{l}\text { Standard met in } \\
\text { subsequent } \\
\text { cycles }(\%)\end{array}$ & $p$ value & $\begin{array}{l}\text { Odds } \\
\text { ratio }\end{array}$ & $\begin{array}{l}95 \% \text { confidence } \\
\text { interval }\end{array}$ \\
\hline \multirow[t]{3}{*}{ High FN risk } & Both indications & 74.4 & 77.8 & 0.298 & 0.30 & $0.91-1.58$ \\
\hline & Lung cancer & 47.0 & 48.3 & 0.869 & 1.05 & $0.65-1.72$ \\
\hline & Breast cancer & 83.0 & 86.2 & 0.298 & 1.28 & $0.88-1.85$ \\
\hline \multirow[t]{3}{*}{ Intermediate $\mathrm{FN}$ risk } & Both indications & 51.3 & 51.1 & 0.948 & 0.99 & $0.85-1.16$ \\
\hline & Lung cancer & 41.0 & 46.1 & 0.168 & 1.13 & $0.98-1.55$ \\
\hline & Breast cancer & 61.3 & 55.9 & 0.139 & 0.80 & $0.64-1.00$ \\
\hline
\end{tabular}

assessment and the guideline plays a decisive role as a subsequent criterion [19].

In breast cancer, the guideline adherence at a high FN risk of $85 \%$ is relatively good and remains the same compared to the preliminary examination. In case of intermediate FN risk, the lack of G-CSF prophylaxis, currently $32.3 \%$ and $33 \%$, respectively, has hardly changed compared to the previous survey. The proportion of over-treated patients with moderate FN risk decreased from 16.6 to $8.8 \%$. Nevertheless, a large proportion of the patients are still treated inadequately.

The quality of therapy needs to be improved by treating patients in tumor centers and organ-specific tumor centers. The German Cancer Society (DKG) has defined criteria for this purpose, which also apply in a similar form to the
Comprehensive Cancer Centers (CCC) in Germany. In addition, the certification of oncology centers is also being carried out by the German Society of Hematology and Medical Oncology (DGHO). It has been shown that the G-CSF prophylaxis guideline adherence is significantly better in these centers. However, this effect is mainly caused by good guideline adherence at breast cancer centers. However, there is also considerable potential to optimize the implementation of guidelines in the certified centers.

Differences were also seen between specialties of oncologists. For example, the guideline adherence of pulmonology institutions was significantly worse than GL adherence at hematology and oncology institutions. So far, there is no explanation for these differences, since the guideline on lung cancer 
Table 3 Number of patients with dose reductions at the second cycle and compliance with guidelines

\begin{tabular}{|c|c|c|c|c|}
\hline & \multicolumn{2}{|c|}{ Dose reduction } & \multicolumn{2}{|c|}{ Standard dose } \\
\hline & $n$ & $\%$ & $n$ & $\%$ \\
\hline \multicolumn{5}{|l|}{ Lung cancer } \\
\hline Yes (with G-CSF) & 42 & 22.5 & 116 & 30.1 \\
\hline Yes (without risk factors) & 6 & 3.2 & 23 & 6.0 \\
\hline Yes (dose reduction without G-CSF) & 58 & 31.0 & 0 & 0.0 \\
\hline Yes (dose reduction, no risk factors, no G-CSF) & 13 & 7.0 & 0 & 0.0 \\
\hline No (overuse: G-CSF without risk factors) & 0 & 0.0 & 9 & 2.3 \\
\hline No (overuse: G-SCF and dose reduction) & 11 & 5.9 & 0 & 0.0 \\
\hline No (underuse: dose reduction and risk factors) & 14 & 7.5 & 0 & 0.0 \\
\hline No (underuse: no G-CSF) & 43 & 23.0 & 238 & 61.7 \\
\hline Gesamt & 187 & 100.0 & 386 & 100.0 \\
\hline \multicolumn{5}{|l|}{ Breast cancer } \\
\hline Yes (with G-CSF) & 10 & 34.5 & 428 & 55.4 \\
\hline Yes (without risk factors) & 0 & 0.0 & 97 & 12.6 \\
\hline Yes (dose reduction without G-CSF) & 4 & 13.8 & 0 & 0.0 \\
\hline Yes (dose reduction, no risk factors, no G-CSF) & 0 & 0.0 & 0 & 0.0 \\
\hline No (overuse: G-CSF without risk factors) & 0 & 0.0 & 36 & 4.7 \\
\hline No (overuse: G-SCF and dose reduction) & 6 & 20.7 & 0 & 0.0 \\
\hline No (underuse: dose reduction and risk factors) & 6 & 20.7 & 0 & 0.0 \\
\hline No (underuse: no G-CSF) & 3 & 10.3 & 211 & 27.3 \\
\hline Total & 29 & 100.0 & 772 & 100.0 \\
\hline
\end{tabular}

from 2010 also contains a short but correct section on G-CSF prophylaxis [20, 21]. In gynecological oncology, guideline adherence was higher than in hematology/oncology, this difference however was statistically not significant.
The guideline-congruent application of G-CSF in the first treatment cycle was not different than in subsequent cycles, which confirms the results of the first study $[12,22]$

At around 10\% less than in the first study, the physicians surveyed believe that $74.8 \%$ of them adhere to the guidelines regularly and $16.2 \%$ in unclear situations, i.e. more than $90 \%$
Fig. 2 Importance of decisionmaking tools for treatment decisions (bottom scale: 1 = "not at all important" to $10=$ "extremely important")

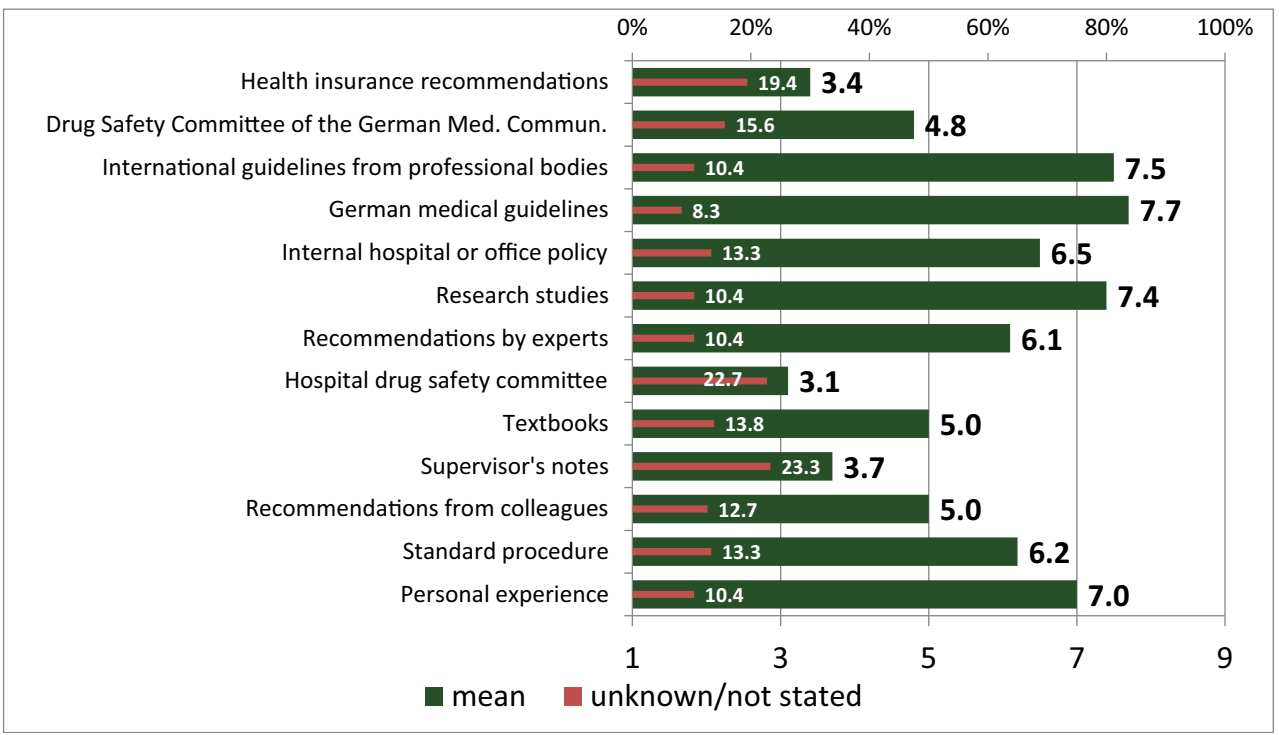


Fig. 3 Activities and contributions of physicians to journals, textbooks, guidelines (in percent)

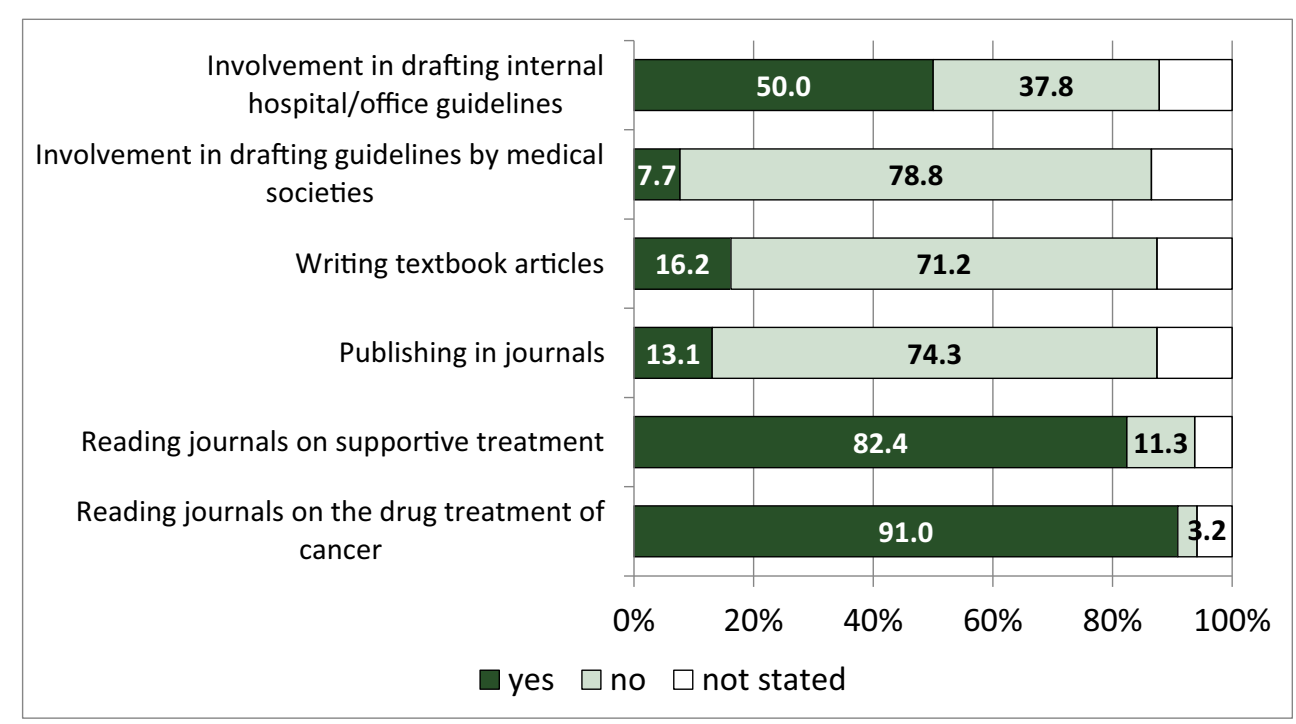

of them attest to their own guideline adherence and willingness to apply the guideline. However, the reality is very different. This discrepancy must be further clarified and reduced.

In contrast to some other countries, cost is not prohibitive to prescribing G-CSF in Germany, as shown by this and the previous study $[12,23]$. In contrast to the previous CART analysis, the duration of the professional experience does not play a role for pulmonologists any more. At that time, professional experience of $\geq 22$ years was associated with reduced GL adherence [12]. The association with congress participation has changed. If two or more international congress visits per year previously differentiated the group with poorer guideline adherence, the visit of two or more national congresses now describes the group with better guideline adherence. The attendance of international congresses is no longer apparent in GL adherence. This could mean that older physicians with longer professional experience and international congress participants no longer play a significant role in the decision to use supportive therapy, or that this group has improved its competence in supportive therapy.

For gynecologists and hematologist-oncologists, the effect of the duration of the education on guideline adherence has

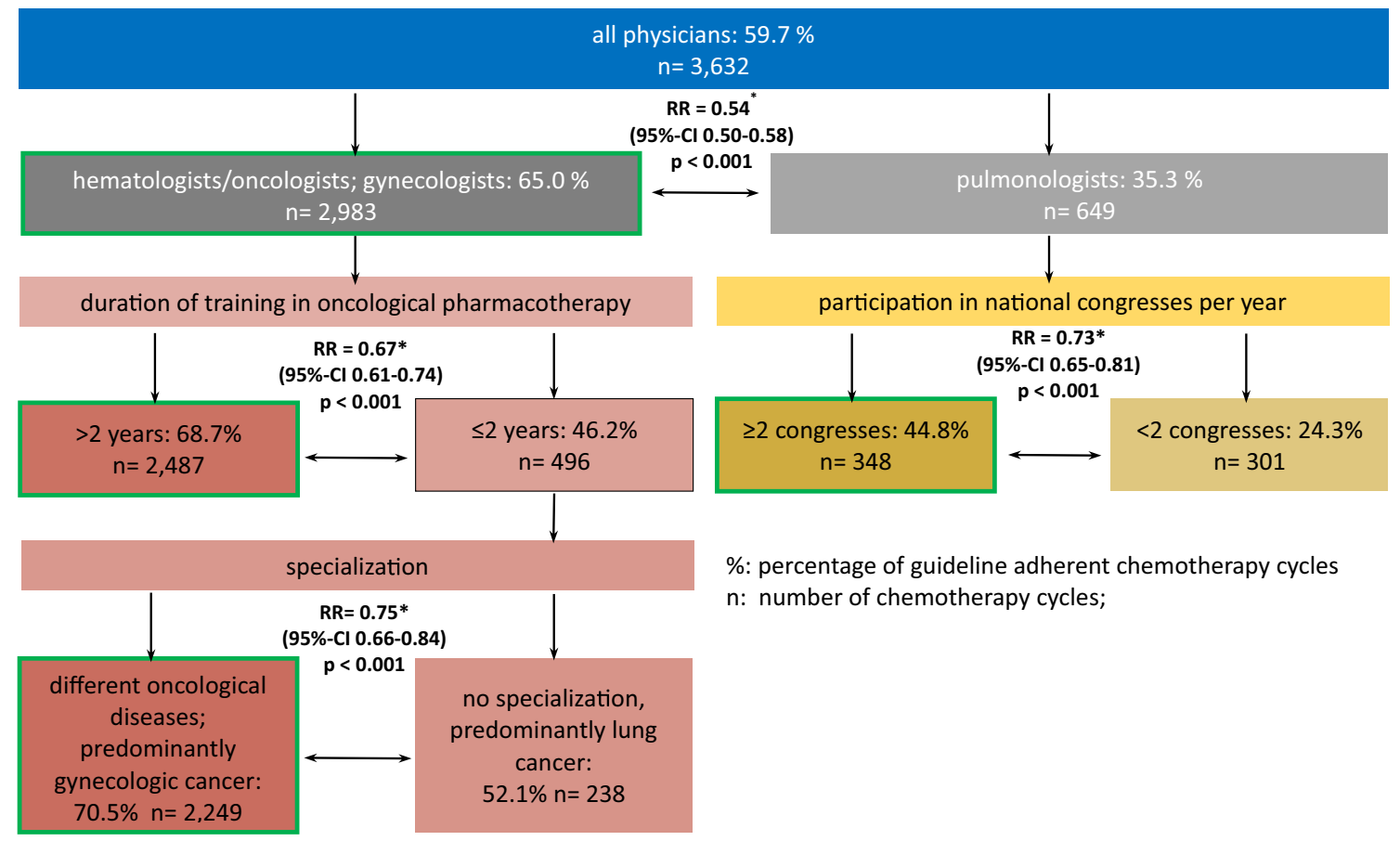

Fig. 4 Guideline-adherent chemotherapy cycles by profile of the physicians; classification and regression tree analysis (CART): a tree-building binary recursive partitioning method, starting with 3632 chemotherapy cycles 
changed. Whereas previously physicians with fewer than 8 years of professional training had better guideline adherence, it is now physicians with more than 2 years of professional training who have better GL adherence. It can be assumed that the group with a long education period, but possibly less patient contact, will now decide less on the supportive therapy.

Overall, it can be seen that G-CSF prophylaxis in patients with lung cancer treated by pulmonologists is the least consistent with the current guidelines, even if adherence has improved compared to the prior examination.

In order to achieve a more comprehensive application of guidelines in Germany, it therefore seems to make sense to promote and optimize training measures which are also urged by the German Institute for Quality and Efficiency in Health Care (IQWiG) [24].

Funding The study was supported by unrestricted grants from Amgen $\mathrm{GmbH}$ and Hexal GmbH to the AIO-Studien-gGmbH of the German Cancer Society, AIO-SUP-0215. The funding source did not have any access to the data and was not involved in data analysis or manuscript writing. Professor Link received research funding and honoraria, acted on speakers' bureau, and/or consulted for Amgen, Chugai, Hexal-Sandoz, Lilly, MSD Oncology, Novartis, Pfizer, Teva, and Vifor Pharma. The authors confirm that they have full control of all primary data and agree to allow the journal to review their data if requested.

\section{Compliance with ethical standards}

Conflict of interest The authors declare that they have no competing interests.

Ethical statement An opinion has been obtained from the Ethics Committee of Rhineland-Palatinate, Mainz, Germany, in accordance with the guidelines and recommendations for ensuring Good Epidemiological Practice (GEP) [25]. Due to the nature of the study, no additional opinions from ethics committees were required. All procedures performed in this study involving human participants were in accordance with the ethical standards of the institutional and/or national research committee and with the 1964 Declaration of Helsinki and its later amendments or comparable ethical standards.

Open Access This article is distributed under the terms of the Creative Commons Attribution-NonCommercial 4.0 International License (http:// creativecommons.org/licenses/by-nc/4.0/), which permits any noncommercial use, distribution, and reproduction in any medium, provided you give appropriate credit to the original author(s) and the source, provide a link to the Creative Commons license, and indicate if changes were made.

\section{References}

1. Dale DC, Crawford J, Klippel Z, Reiner M, Osslund T, Fan E,

Morrow PK, Allcott K, Lyman GH (2018) A systematic literature review of the efficacy, effectiveness, and safety of filgrastim. Support Care Cancer 26(1):7-20
2. Lyman GH, Michels SL, Reynolds MW, Barron R, Tomic KS, Yu J (2010) Risk of mortality in patients with cancer who experience febrile neutropenia. Cancer 116:5555-5563

3. Lyman GH, Dale DC, Culakova E, Poniewierski MS, Wolff DA, Kuderer NM, Huang M, Crawford J (2013) The impact of the granulocyte colony-stimulating factor on chemotherapy dose intensity and cancer survival: a systematic review and meta-analysis of randomized controlled trials. Ann Oncol 24(10):2475-2484

4. Smith TJ, Bohlke K, Lyman GH, Carson KR, Crawford J, Cross SJ, Goldberg JM, Khatcheressian JL, Leighl NB, Perkins CL, Somlo G, Wade JL, Wozniak AJ, Armitage JO, American Society of Clinical Oncology (2015) Recommendations for the use of WBC growth factors: American Society of Clinical Oncology clinical practice guideline update. J Clin Oncol 33(28):3199-3212

5. Crawford J, Caserta C, Roila F, Group ObotEGW (2010) Hematopoietic growth factors: ESMO clinical practice guidelines for the applications. Ann Oncol 21(suppl 5):v248-vv51

6. Crawford J. (2014) NCCN® Practice Guidelines in Oncology v.2.2014; Myeloid growth factors. J Natl Compr Canc Netw [Internet]. 01/04/2015. Available from: http://www.nccn.org/

7. Vehreschild JJ, Bohme A, Cornely OA, Kahl C, Karthaus M, Kreuzer KA et al (2014) Prophylaxis of infectious complications with colony-stimulating factors in adult cancer patients undergoing chemotherapy-evidence-based guidelines from the infectious diseases working party AGIHO of the German Society for Haematology and Medical Oncology (DGHO). Ann Oncol 25(9): $1709-1718$

8. Aapro MS, Bohlius J, Cameron DA, Dal LL, Donnelly JP, Kearney $\mathrm{N}$ et al (2011) 2010 update of EORTC guidelines for the use of granulocyte-colony stimulating factor to reduce the incidence of chemotherapy-induced febrile neutropenia in adult patients with lymphoproliferative disorders and solid tumours. Eur J Cancer 47(1):8-32

9. Jordan K, Feyer P, Höller U, Link H, Wörmann B, Jahn F (2017) Supportive treatments for patients with cancer. Dtsch Arztebl Int 114(27-28):481-487

10. Bennett CL, Djulbegovic B, Norris LB, Armitage JO (2013) Colony-stimulating factors for febrile neutropenia during cancer therapy. N Engl J Med 368(12):1131-1139

11. Bokemeyer C, Gascon P, Aapro M, Ludwig H, Boccadoro M, Denhaerynck K et al (2017) Over- and under-prophylaxis for chemotherapy-induced (febrile) neutropenia relative to evidencebased guidelines is associated with differences in outcomes: findings from the MONITOR-G-CSF study. Support Care Cancer 25(6):1819-1828

12. Link H, Nietsch J, Kerkmann M, Ortner P, Supportive Care Group of the German Cancer S (2016) Adherence to granulocyte-colony stimulating factor (G-CSF) guidelines to reduce the incidence of febrile neutropenia after chemotherapy-a representative sample survey in Germany. Support Care Cancer 24(1):367-376

13. Benjamini Y, Hochberg Y (1995) Controlling the false discovery rate: a practical and powerful approach to multiple testing. J R Stat Soc Ser B Methodol 57(1):289-300

14. Schulz KF, Grimes DA (2005) Multiplicity in randomised trials I: endpoints and treatments. Lancet 365(9470):1591-1595

15. von Elm E, Altman DG, Egger M, Pocock SJ, Gøtzsche PC, Vandenbroucke JP, for the STROBE Initiative (2007) The strengthening the reporting of observational studies in epidemiology (STROBE) statement: guidelines for reporting observational studies. PLoS Med 4(10):e296

16. Breiman L, Friedman JH, Olshen RA, Stone CJ (1984) Classification and regression trees. Chapman and Hall, New York

17. Breiman L (2001) Statistical modeling: the two cultures (with comments and a rejoinder by the author). Stat Sci 16(3):199-231

18. Lyratzopoulos G, Liu MP-H, Abel GA, Wardle J, Keating NL (2015) The association between fatalistic beliefs and late stage at 
diagnosis of lung and colorectal cancer. Cancer epidemiology, biomarkers \& prevention: a publication of the American Association for Cancer Research, cosponsored by the American Society of Preventive Oncology 24(4):720-726

19. Freyer G, Kalinka-Warzocha E, Syrigos K, Marinca M, Tonini G, Ng SL, Wong ZW, Salar A, Steger G, Abdelsalam M, DeCosta L, Szabo Z (2015) Attitudes of physicians toward assessing risk and using granulocyte colony-stimulating factor as primary prophylaxis in patients receiving chemotherapy associated with an intermediate risk of febrile neutropenia. Med Oncol 32(10):236

20. Goeckenjan G, Sitter H, Thomas M, Branscheid D, Flentje M, Griesinger F et al (2011) Prevention, diagnosis, therapy, and follow-up of lung cancer. Interdisciplinary guideline of the German Respiratory Society and the German Cancer Societyabridged version. Pneumologie 65(8):e51-e75

21. Goeckenjan G, Sitter H, Thomas M, Branscheid D, Flentje M, Griesinger F, Niederle N, Stuschke M, Blum T, Deppermann KM, Ficker J, Freitag L, Lübbe A, Reinhold T, Späth-Schwalbe E, Ukena D, Wickert M, Wolf M, Andreas S, Auberger T, Baum R, Baysal B, Beuth J, Bickeböller H, Böcking A, Bohle R, Brüske I, Burghuber O, Dickgreber N, Diederich S, Dienemann H, Eberhardt W, Eggeling S, Fink T, Fischer B, Franke M, Friedel G, Gauler T, Gütz S, Hautmann H, Hellmann A, Hellwig D, Herth F, Heußel C, Hilbe W, Hoffmeyer F, Horneber M, Huber R, Hübner J, Kauczor HU, Kirchbacher K, Kirsten D, Kraus T, Lang S, Martens U, MohnStaudner A, Müller KM, Müller-Nordhorn J, Nowak D, Ochmann U, Passlick B, Petersen I, Pirker R, Pokrajac B, Reck M, Riha S,
Rübe C, Schmittel A, Schönfeld N, Schütte W, Serke M, Stamatis G, Steingräber M, Steins M, Stoelben E, Swoboda L, Teschler H, Tessen H, Weber M, Werner A, Wichmann HE, Irlinger Wimmer E, Witt C, Worth H (2011) Prevention, diagnosis, therapy, and followup of lung cancer: interdisciplinary guideline of the German Respiratory Society and the German Cancer Society. Pneumologie 65(1):39-59

22. Aarts MJ, Peters FP, Mandigers CM, Dercksen MW, Stouthard JM, Nortier HJ, van Laarhoven HW, van Warmerdam LJ, van de Wouw AJ, Jacobs EM, Mattijssen V, van der Rijt CC, Smilde TJ, van der Velden AW, Temizkan M, Batman E, Muller EW, van Gastel SM, Borm GF, Tjan-Heijnen VCG (2013) Primary granulocyte colonystimulating factor prophylaxis during the first two cycles only or throughout all chemotherapy cycles in patients with breast cancer at risk for febrile neutropenia. J Clin Oncol 31(34):4290-4296

23. Barnes G, Pathak A, Schwartzberg L (2014) G-CSF utilization rate and prescribing patterns in United States: associations between physician and patient factors and G-CSF use. Cancer Med 3(6):14771484

24. IQWIG (2016) Implementation of guidelines - obstructive and beneficial factors. In: (IQWiG) IfQaEiHC (ed) Executive summary of final report V12-04 Version 1.0 Implementation of guidelines, 9 May 2016 edn. IQWIG, Cologne, p. 15

25. Bellach, BM. Bundesgesundheitsbl - Gesundheitsforsch Gesundheitsschutz (2000) 43: 468-475. https://doi.org/10.1007/ s001030070056 